strike without warning. Our hospital, Tan Tock Seng Hospital, has been the national screening centre for severe acute respiratory distress syndrome (SARS) and H1N1 outbreaks in 2003 and 2009. Furthermore, our ED has managed casualties from mass food poisoning in the community. We would like to share our experiences in training our staff for MCI. For the ED to operate smoothly in a MCI, comprehensive training of staff during "peace" time is essential. We have a selected team of doctors and nurses as the department disaster workgroup. This team, together with the hospital emergency planning department, prepare the disaster protocols using an "all hazard approach concept" and aim to minimise variations between different protocols (Conventional, Infectious disease, Hazmat, Radioactive MCI). These protocols are updated regularly, with new information disseminated to all staff. Next, all staff must be well-versed in the protocols. New staffs undergo orientation programmes to familiarize them with the work processes. Regular audits are conducted to ensure that the quality is wellmaintained. Additionally, training also occurs at the interdepartmental and national levels. There are regular activation exercises to test inter-departmental response to $\mathrm{MCI}$ and collaborations with Ministry of Health to conduct disaster exercises e.g. the biennial Kingfisher Exercise in preparation for radiation-related MCI. Such exercises improve communication and working relationships within the ED and with other departments. The camaraderie developed can act as a pillar of support during stressful times of MCI. Lastly, the ED staffs attend local and international courses and conferences to update ourselves on the latest training and knowledge in the handling of MCI. This allows us to share our ideas and to learn from our local and international counterparts, and helps better prepare ourselves.

Prehosp Disaster Med 2011;26(Suppl. 1):s110-s111

doi:10.1017/S1049023X11003700

(P1-39) Support for Functionally Insufficient Key Disaster Hospitals by Dmat for Large-Scale Disaster in Japan T. Kodama, ${ }^{1}$ H. Ohara,${ }^{1}$ D. Jinbo, ${ }^{1}$ H. Shiozawa, ${ }^{1}$ T. Fujino, ${ }^{1}$ N. Motodate, ${ }^{1}$ T. Wada,${ }^{1}$ J. Matsumoto, ${ }^{2}$ Y. Taira ${ }^{2}$

1. Comittee of Dmat, Kawasaki-City, Japan

2. Department of Emergency and Critical Care Medicine, Kawasaki-City, Japan

Background: Japanese Disaster Medical Assistance Team (DMAT) has important duties to support and reinforce functionally insufficient key disaster hospitals in the large-scale disasters. However, it is difficult for Japanese DMAT to fulfill these duties in the current circumstances, because the DMAT consists of individual institutions and Teams have less experience to work in the other institutions. To resolve the issues, disaster drills for some DMATs which consist of several institutions have been held since 2008. We evaluated the effectiveness of the drills and educational system.

Methods: Investigation was performed by surveillance questionnaires to 129 participants in the drill (staffs from DMATs, Red Cross and local government, and sham patients). The questionnaires are: A. Was the cooperative medical practice performed smoothly? B. Was the support for hospitals conducted effectively?, C. Was the medical record for wide-area transportation completed? (for our staffs only), D. Was our activity enough? (for participants excluding us), and $\mathrm{E}$. Was the drill beneficial?

Results: 85 participants including 30 staffs in our institution answered the questionnaires (65.9\%). The number of participants who answered 'Yes' was as follows: A. 48 (56.5\%), B. 64 (75.3\%), C. 8 (26.7\%), D. 44 (83.0\%) and E. 81 (95.3\%).

Discussion: Although the cooperative medical practice was not so smooth, it was recognized that members in DMATs got mutual trust by their high knowledge and skills. And it was suggested that DMAT could not command and control the hospital staffs without their understanding the equipments and documentations used by DMAT. And it is ideal to change the mindset of hospital top managements towards the disaster medicine through the repeated drills.

Conclusion: The repeated disaster drills at the local area is essential to make DMAT function sufficiently.

Prehosp Disaster Med 2011;26(Suppl. 1):s111

doi:10.1017/S1049023X11003712

\section{(P1-40) Development of Hospital Triage Training at the Chris Hani Baragwanath Hospital \\ F. Plani}

Trauma Unit, Department of Surgery, Johannesburg, South Africa

Development of Hospital Triage Training at the Chris Hani Baragwanath Hospital F. Plani ${ }^{1}$, E Degiannis, P Lingham No disaster training had ever been carried out at the $\mathrm{CHBH}$, the biggest hospital in the world with over 2900 beds and over 5000 staff members. The 2010 Disaster Plan required that all clinical staff undergo the appropriate training. Basic Interprofessional Training for Trauma Disasters 411 staff members attended a half day course during May/June 2010, presented and sponsored by $\mathrm{CHBH}$ Trauma Directorate consultants. The aims of the course were to disseminate the trauma disaster plan in interdisciplinary and interprofessional fashion, clarify hospital triage, familiarize staff with Trauma Unit equipment, and practice resuscitations in mass casualty incidents. The course started with presentations on $\mathrm{CHBH}$ Disaster Plans and Protocols and Principles of Triage and Standard of Care in Disasters. This was followed by 3 multiple patient scenarios with rotating groups, over a whole hospital floor with: 1) 50 mixed patients outside the hospital; 2) 10 serious patients in the ER; 3) 10 patients deteriorating later in a ward or ICU. Next were individual patient resuscitations, Primary, Secondary, Tertiary Survey and "hand-over", patient resuscitations in a disaster, using the contents from "Disaster Bags" and questionnaires to assess confidence and suitable treatment areas (Red, Yellow, Green, OT) for allocation in a disaster. The course was wrapped up by a familiarization visit to ED and the colour coded areas.

Results: Final questionnaires demonstrated that all participants were a lot more confident in the triage of patients and the initial resuscitation using the implements found in the resuscitation room and the "Disaster Bags". The course has been adopted as part of staff orientation at all professional levels from 2011 onwards, and is in the process to be extended to secondary level hospitals in Gauteng, RSA.

Prehosp Disaster Med 2011;26(Suppl. 1):s111

doi:10.1017/S1049023X11003724 